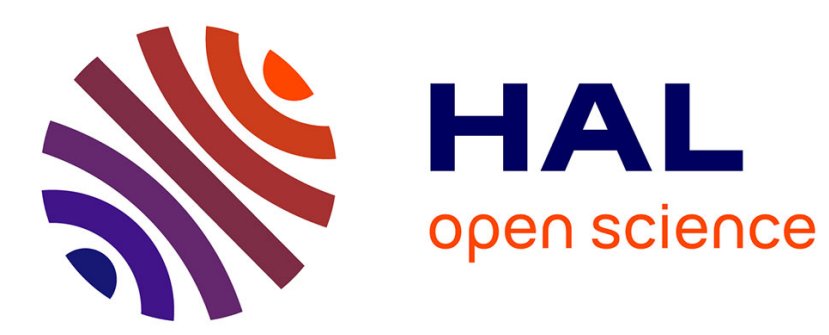

\title{
L'interprétation du diagramme eutectoïde Fe-Fe3C, doit-elle être modifiée?
}

P. Dejean

\section{To cite this version:}

P. Dejean. L'interprétation du diagramme eutectoïde Fe-Fe3C, doit-elle être modifiée?. J. Phys. Radium, 1935, 6 (12), pp.525-536. 10.1051/jphysrad:01935006012052500 . jpa-00233376

\section{HAL Id: jpa-00233376 https://hal.science/jpa-00233376}

Submitted on 1 Jan 1935

HAL is a multi-disciplinary open access archive for the deposit and dissemination of scientific research documents, whether they are published or not. The documents may come from teaching and research institutions in France or abroad, or from public or private research centers.
L'archive ouverte pluridisciplinaire HAL, est destinée au dépôt et à la diffusion de documents scientifiques de niveau recherche, publiés ou non, émanant des établissements d'enseignement et de recherche français ou étrangers, des laboratoires publics ou privés. 


\title{
L'INTERPRÉTATION DU DIAGRAMME EUTEGTOIDE Fe-Fe ${ }^{3}$ C, DOIT-ELLE ÊTRE MODIFIÉE?'
}

Par P. DEJEAN.

Professeur à la Faculté des Sciences de Grenoble.

\begin{abstract}
Sommaire. - Les diagrammes expérimentaux invoqnés par M. J. Seigle dans un précédent article (J. Physique, 1934, t. 5, p. 37) ne paraissent pas à M. Dejean ćtablis dans des conditions suffisamment sûres (barreaux étudiés trop courts refroidissements trop rapides; pour modifier la théorie classique des transformalions du fer et des aciers.

Réponse de M. Seigle, et note additionnelle de M. Dejean.
\end{abstract}

M. Seigle a publié, dans le Jourual de Physique et dans diverses autres revues, une série d'articles $\left(^{1}\right)$ qui arrivent tous à cette conclusion, que :

L'interprétation classique du diagramme ( $\mathrm{Fe}-\mathrm{Fe}^{3} \mathrm{C}$ ), dans la région eutectoïde, ne cadre pas avec certaines particularités des observations micrographiques, dilatométriques et magnétiques qu'il a faites. Il la considère donc comme inexacte et il en propose une autre.

Toute son argumentation repose sur deux propositions fondamentales :

La première est relative à la transformation allotropique $\alpha \Longleftarrow \gamma$ du fer pur.

La deuxième intéresse la perlite, sa nature et son mode de formation.

Ces deux propositions sont assez graves pour mériter un examen un peu détaillé. C'est ce que nous allons essayer de faire.

(1) Nouvelle théorie proposée ponr la formation des aciers recuils Le Géne Cirll, 14 et 21 octobre 1933,103 , p. 369 et 4110.

Quelques aspects micrographiques d'un acier ordinaire dur au carbone recuit Chinie et Industrie, décembre 1933, 30, p. 1182.

Etude sur les changøments magnétiques dans le fer et les aciers en fonction de la température. Journal de Physiqne, Série VIl janvier 193', 1. 5, p 37.

La transformation $\alpha \vec{\forall} \gamma$ dans les aciers, envisagée au point de vue de ses effets dilatométrique et magnétiques Chimie et Intustrie février 193ł, l 31, p. 282 .

Diagramme proposé pour la représentation des a iers au carbone. Chime el Industrie, avril 193', t. 31, p. 7:3.

Structures perliliques diverses d'un acier à 0,22 de carbone Industrie minercte $1^{\mathrm{e}} \mathrm{mai} 1934$.

Un nouveau diagramme fer carbone pour les aciers et quelques unes de ses conséquences Le Génie Civil 19 et 26 mai 1954 ,

Critique du diagramme entectroïde fer cémentite et nouveau diagramme propose Congres de Fonderie de Nancy (juillet 1934). Bulletin de l'Associalion technique de fonderie juillet 193. t. 8, p. 289.

Textures micrographiques et duretés d’un acier orisinaire à 0 85 de carbone Chimie ei Industrie, septembre 193k, 1. 32 p. 50 5 . Etude d'un acier ordinaire à 0,58 de carbone, Chimie et Industie, octobre $193 \dot{4}$, l 32 , p $i$ i

Texture de transition entre zone trempée et non trempée dans un acier à 0,22 de carbone. Chimie et Industrie, novembre 1934, t 32, p. 1039 .
1. La transformation allotropique $\alpha \Longleftarrow \gamma$ dans le fer pur. - A ce sujet, M. Seigle écrit( $\left.{ }^{1}\right)$ :

"Une série d'essais nicrographiques et dilatométriques, ainsique les essais dilatomagnétiques relatés dans cette étude (') m'ont condu tu l'hypothèse ci-après: a) Le domaine du fer a pur ne dépasse pas 710 à $720^{\circ}$ (point critique $\left.A_{1}\right) ; b$ ) Le domaine du fer $\gamma$ reste celui qui est au delà de $910^{\circ}$ point $\left.A_{3} ; c\right)$ Dans le domaine intermédiaire de $\mathrm{A}_{1}$ à $\mathrm{A}_{3}$ existe un état mixte $\alpha \beta$, à proportions décroissantes de $\alpha$ au chauffage, et à proportions décroissantes de $\because$ au refroidissement. Il n'y a pas lieu de considérer un élat $\beta$.

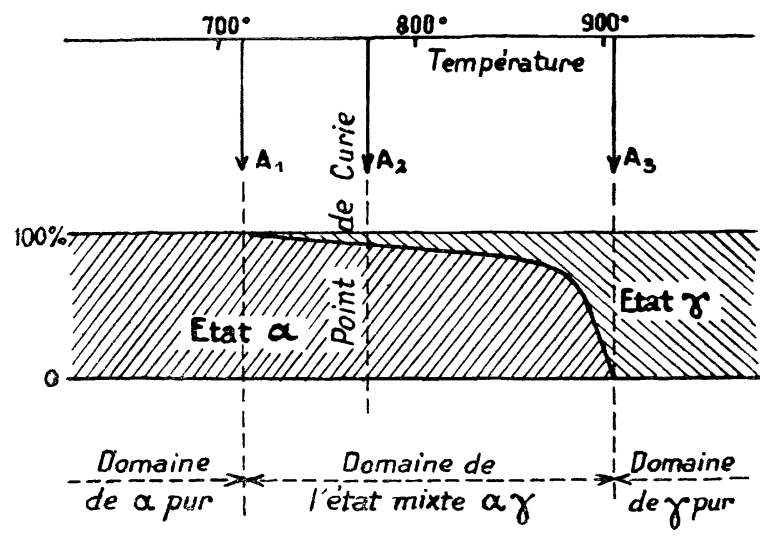

Fig. 1. - Zone de (ransformation allotropique du fer pur. (D'après J. Seigle.)

“ Les proportions respectives des deux états a et $\gamma$ varient en fonction de la température à l'échauffement dans le sens qu'indique la figure 1 ; on voil que la proportion de a reste très grande jusque vers $825 \dot{a}$ $850^{\circ}$, et le gros de la transformation $\alpha \rightarrow \gamma$ se fait ensuite dans un intervalle de température de $75 \dot{a} 50^{\circ}$ ou

(') Seigle. Journal de Physique, janvier 1934. page 46, $2 \cdot$ colonne, ligne 4. 
quelquefois bien moins. Cet étalement en température est très variable d'un fer ou acier extra-doux, à un autre.

"Au refroidissement, on a une allure du même genre, compte tenu du " retard » $\grave{a}$ la transformation dans le sens $\gamma \rightarrow \alpha$. 》

Il faut retenir de cette cilation que :

$A$. C'est à la suite d'essais micrographiques dilatométriques et dilatomagnétiques que M Seigle a établi ses conclusions.

B. La transformation $\alpha \Longleftarrow \gamma$ est étalée en température et cet étalement est très variable d'un fer ou acier extra-doux à un autre.

$C$. Dans le domaine intermédiaire de $\mathbf{A}_{1}$ à $\mathbf{A}_{3}$, il existe un état mixte $\alpha \gamma$.

Nous allons examiner successivement ces trois points.

A. Essais Micrographiques et dilatométriques. Malgré nos recherches, nous n'avons pu retrouver dans les diverses publications précédemment notées aucune étude micrographique sur le fer pur. En revanche, cet auteur, nous donne (Journal de Physique, janvier 1934, page 39) un diagramme dilatométrique relatif au fer électrolylique et fer ARMCO qui est un fer à peu près pur. Nous reproduisons figure 2 ce diagramme.

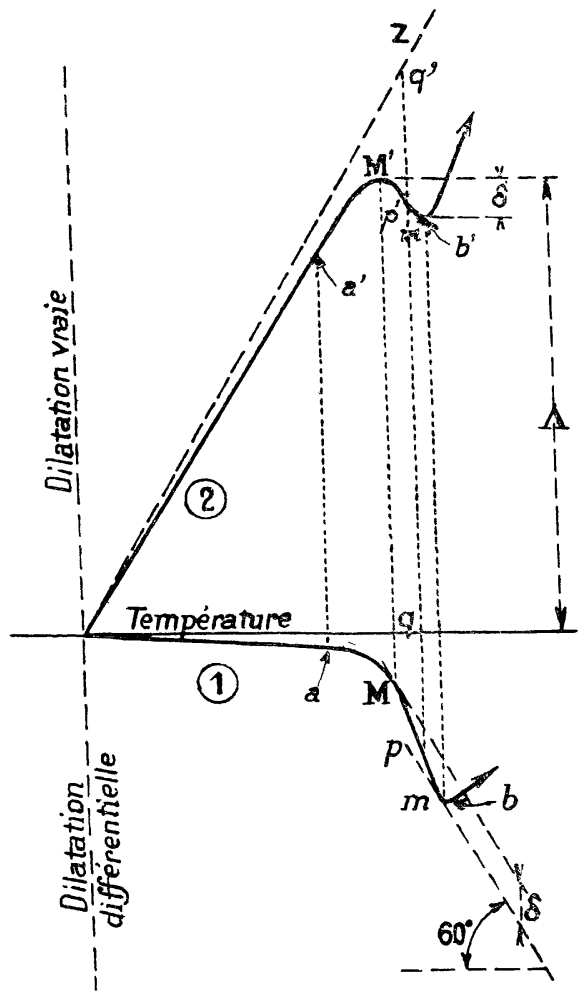

Fig. 2. - Diagrammes pour du fer électrolytique et du fer ARMCO. (D'après J. Seigle.)

B. Etalement de la transformation $\alpha=\gamma$. Le fait de mettre sur le même pied le fer pur et les aciers extra-doux constitue une tendance assez regrettable, car nous montrerons plus loin (fig. 11) qu'il y a une très grosse différence entre la courbe dilatométrique du fer pur et celle de l'acier extra-doux. Or, si le diagramme de M. Seigle (fig. 2) s'accorde assez bien avec celui d'un acier extra-doux, il est en contradiction très nette avec ceux obtenus par divers auteurs sur des fers très purs dont les analyses sont exactement indiquées.

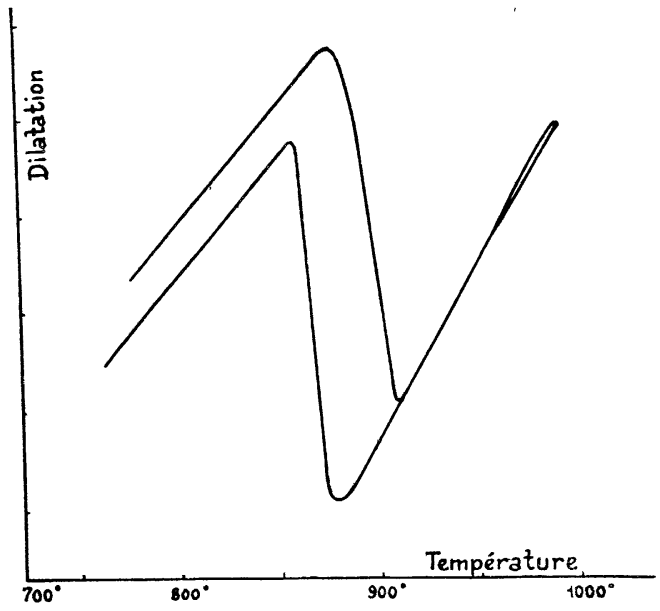

Fig. 3. - Courbe de dilatation d'un fer Electrobévé, dont la composition en pour 100 est donnée ci-après :

$$
\begin{aligned}
& \mathrm{C}=0,0028 ; \mathrm{Si}=0,1446 . \\
& \mathrm{S}=0,0053 ; \quad \mathrm{P}=0,025 . \\
& \mathrm{Cu}=0,097 ; \text { Fe par différence. } \\
& \text { (D'après A. Bouchayer) }
\end{aligned}
$$

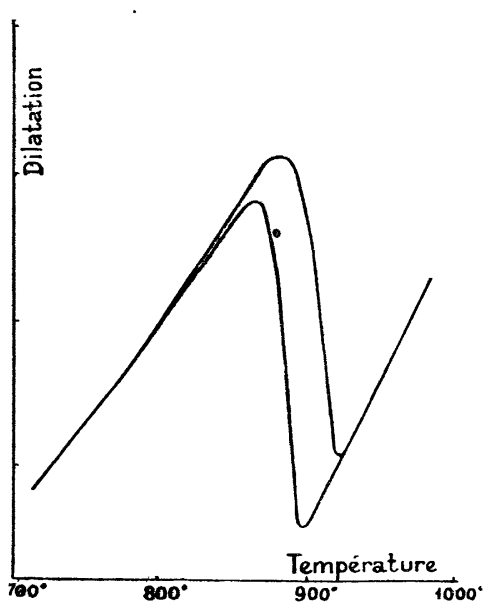

Fig. 4. - Courbe de dilatation d'un fer électrolytique dont la composition pour 1110 est donnée ci-après : $\mathrm{C}=0,006 ; \mathrm{Si}$ : pas de traces.

Mn : traces; $\mathrm{S}=0,006$

$\mathrm{P}$ : pas traces; Cu : pas de traces. (D’après Seikichi Sato.)

C'est ainsi qu'on trouvera figure 3 la courbe de dilatation du fer électrolytique Bévé (extraite d'une brochure publiée en 1920 par les Etablissements Bou- 
chayer et Viallet) $\left(^{1}\right.$ ) et figure 4 celle donnée par Seikichi Sato dans un article sur les recherches dilatométriques des transformations $\mathrm{A}_{3}$ et $\mathrm{A}_{4}$ dans le fer $\operatorname{pur}\left({ }^{2}\right)$.

Les courbes obtenues par ces deux auteurs ont des allures identiques; mais elles diffèrent complètement de celle donnée par M. Seigle.

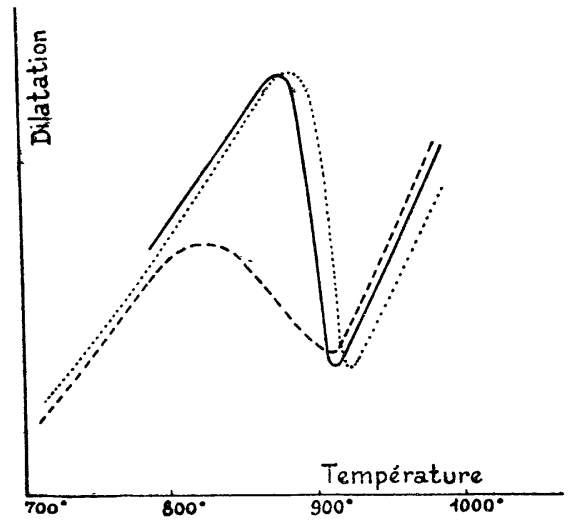

Fig. 5. - Courbe de A. Bouchayer (trait plein). Courbe de Seikichi Sato (trait pointillé). Courbe de J. Seigle (trait interrompu).

Pour miéux noter cette différence, nous avons reproduit à la mème échelle (fig. 亏̆) les courbes de chauffage publiées par les trois auteurs précités. Tandis que celles de Bouchayer el de Sato, qui coïncident sensiblement dénotent une contraction brusque et à peu près isotherme au point $\mathrm{Ac}_{3}$, celle de M. Seigle (qui se rapporte vraisemblablement à un acier extra-doux et non à $d u$ fer pur) est assez étalée.

Je dis à peu près isotherme, car la contraction s'étend sur quelques degrés dans les courbes de Bouchayer et de Sato. Cela peut être attribué :

$1^{\circ}$ Aux quelques impuretés des fers utilisés qui, bien que très purs, ne sont pourtant pas chimiquement purs; $2^{\circ}$ à ce que les vitesses de chauffage et de refroidissement ne sont pas assez lentes pour que l'équilibre thermique soit toujours atteint et $3^{\circ}$ à ce que l'inertie des appareils peut jouer aussi un certain rôle.

Chevenard et Portevin ont donné ( $\left.{ }^{3}\right)$ eux aussi un diagramme dilatométrique relatif à un fer de Suède presque dépourvu de carbone $(\mathrm{C}=0,035$ pour 100$)$, qui donne une anomalie de dilatation au point $A_{3}$ aussi brusque que celle indiquée par $\mathrm{A}$. Bouchayer et par Seikichi Sato.

(1) Adgusta Rodghayer. Quelques propriétés du fer Electrobévé édition J. Rey Grenoble 1920.

(2) Srrmichi SAto. Dilatometric Investigation of the $\mathbf{A}_{3}$ and $\mathbf{A}_{4}$ transformatrons in pure Iron, Science Reports of the Toholu Imperal University, décembre 1925, Série 1, vol. 14, $N \cdot 5$.

(3) Chmprard et Portevin. Revue de Métallurgie, 1925, 22, p. 361, fig. 3 .
Pierre Curie (1), Weiss et Fö̈x $\left(^{(2)}\right.$ de mème que Kotaro Honda ( $\left.{ }^{3}\right)$ ont noté une variation brusque de la susceptibilité magnétique du fer pur lorsqu'on franchit le point $A_{3}$. Il serait bien étonnant que tous ces éminents auteurs se soient trompés et aient noté un phénomène assez brusque alors que d'après $M$. Seigle, il aurail dù être étalé depuis $\Lambda_{1}$ jusqu'à $A_{3}$ et tout au moins assez graduel entre $850^{\circ}$ et $900^{\circ}$.

C. En're $A_{1}$ et $A_{3}$ il existe un état mixte $\alpha \%$ Puisque l'examen aux rayons $X$ montre que dans cet intervalle nous avons affaire à un métal cristallisé, l'état mixte $\alpha$ Y ne pourrait être réalisé que par des cristaux mixtes.

Sans vouloir aborder ici l'objection qui pourrait être soulevée par la difficulté d'obtenir des cristaux mixtes de fer $\alpha$ (cristaux cubiques centrés) et de fer $\gamma$ (cristaux cubiques à faces centrées) $\left({ }^{4}\right)$ nous dirons que les données expérimentales de M. Seigle ne sont pas assez convaincantes pour que nous essayons de le suivre sur un terrain aussi peu sûr.

2. La perlite est-elle un eutectoïde? - Abordons maintenant la deuxième proposition, celle relative à la perlite.

On sait que, dans ce que M. Seigle appelle la théorie classique des aciers recuits, la perlite est considérée comme un eutectoïde de fer et de carbure de fer. Or cet auteur se propose de montrer qu'il n'en est rien, et que la perlite n est qu'un pseudo-eutectoïde. Il a fait un certain nombre de griefs à la théorie classique que nous avons groupés sous forme de trois objections principales Nous allons les étudier successivement.

$1^{\mathrm{re}}$ Objection. - La perlite n'est pas un eutectoide, sa teneur en carbone pouvant varier de 0,9 à 0,6 pour 100 environ. - Cette opinion de M. Seigle qui n'est étayée que sur des observations micrographiques semble provenir d'une confusion. Le diagramme d'état du système $\mathrm{Fe}-\mathrm{F}^{3} \mathrm{C}$, aussi bien dans la région eutectoïde que dans toute autre région, est un diagramme d'équilibre, c'est-à-dire qu'il suppose que les variations thermiques ont été assez lentes pour que les constituants formés soient des constituants d'équilibre. C'est justement ce dont M. Seigle ne semble pas avoir suffisamment tenu compte. Il s'appuie en effet sur une remarque de MM. Guillet et Portevin $\left(^{5}\right)$, qui spécifie que, pour évaluer la teneur en carbone des aciers

(1) P. Cunie. Propriétés magnétiques des corps à diverses températures. Annales de Chimie et de Physique 1895, 7e S., t. 5 , p. 289 .

(2) Weiss et Foex. Etude de l'aimantation des corps ferromagnétiques au dessus du point de Curie. Journal de Physique, 1911, วe S., t 1, p. 745 .

$\left.{ }^{3}\right)$ Kotaro Honda. Does the Gritical Point depend of the Strength of the Magnetizing Field? Science Reports of the Tônoku Impérial Untversity 1922. vol. 10, No 6, p. 433.

(4) Voir à ce sujet l'opinion de H. Weiss. Revue de métallurgie 1925, t. 22 , p. $341,2^{\text {e }}$ colonne.

(5) Guillet et Pontevin. Précis de Métallographie (édition 1924), page 160 , Dunod, éditeur. 
d'après la surface occupée par la perlite, il faut toujours opérer sur des aciers recuits dans les mêmes condilions et que notamment, la vitesse de refroidissement agit sur la ségrégation de la ferrite proeutectoïde, de sorte que l'augmentalion de la vitesse de refroidissement tend à diminuer la surface de la ferrile.

Or, dil M. Seigle, la diminution de la surface occupée par la ferrite entraîne laugmentation de celle occupée par la perlite et alors le carbone total de l'acier étant resté le même, il faut lonc en conclure que lon aura de la perlite dont la teneur moyenne en carbone est inférieure à 0,9 pour 100 .

Cette conclusion n'est pas exacte pour la raison précédemment énoncée. Lorsque le refroidissement n'est pas suffisamment lent, ce n'est pas une perlite d'équi. libre à 0,9 pour 100 de carbone que l'on a; mais un aspect perlitique qui peut aller jusqu'à la troostite lorsque la vitesse de refroidissement est assez grande. Ne trouve-t-on pas, non sans étonnement du reste, dans des dessins micrographiques de M. Seigle (1), de la perlite voisinant avec de la martensite! Ce n'est donc pas sur de tels aspects que l'on peut raisonner pour dire que la perlite n'est pas un entectoïde, car il est bien évident qu'il y a, dans ce cas, confusion entre la perlite et la troostite.

Mais M. Seigle va plus loin. Il ajoute ceci : “ Jai constaté en outre qu'un refroidissement lent normale mais effectué après un chaulfage à température élevée $\left(100 \%^{\circ}\right.$ et plus) diminue aussi la surface de la ferrite libre (toujours dans l'acier étudié à 0,58 pour 100 de carbone). )

En se reportant à l'étude à laquelle M. Seigle fait allusion $\left({ }^{2}\right)$, on constate que, ce qu'il appelle refroidissement lent normal correspond à une duréc de passage $\mathrm{Ar}_{3}$ à $\mathrm{Ar}_{1}$ de l'ordre de 3 à 15 ou 20 minutes.

Une telle définition de la vitesse de refroidissement est assez vague. et imprécise. mais on peut dire qu'il ne s'agit pas là d'un refroidissement très lent. Dans un tel acier, comme dans toule autre nuance, la perlite d'équilibre (la seule à laquelle se référe le diagramme) a bien une composition très voisine de 0,9 pour 100 de carbone. Le fait qu'on peut modifier cette composition par un traitement approprić tout en conservant un aspect plus ou moins vaguement perlitique n'infirme en rien ce résultat.

On peut simplement retenir de l'expérience précitée (chauffage à $1000^{\circ}$ et plus) que le retour à l'état d’équilibre est d'autant plus difficile que le chauffage préalable a été fait à température plus élevée. La trempe des aciers à outils rapides illustre ce fait d'une manière particulièrement frappante.

2. Objection. - Au chauffage, la perlite ne se transforme pas en solution solide au point $.1 c_{1}$; mais seule-

(1) J. Seiglk. Texlures micrographiques et duretés d'un acier ordinaire à 0,85 pour cent de carbone. Chime et Industrie, sept + mbre $19: 34$, vol. 32 , fig. $\delta$, page 513 et fig. 10 p 515.

(2) J. Seigre. Quelques aspects micrographiques d'un acier ordinaire dur au carbone recuit. Chimie et Industrie, décembre 1933, p. 1282. ment lorsqu'il s'est formé une assez forte proportion de fer $\gamma, c$ 'est-à-dire vers $.1 c_{3}$.

De même, au refroidissement, le fer commence à se transformer de fer ${ }_{i}$ en fer $\alpha$, au point $\mathrm{Ar}_{3}$. Dès que la proportion de fer a augmente tant soit peu, la cémentile ne peut plus rester en solution : il y a précipitntion de cette cémentite qui cherche a s'isoler ; mais elle entraîne en même temps de la ferrite, d'où formation de perlite.

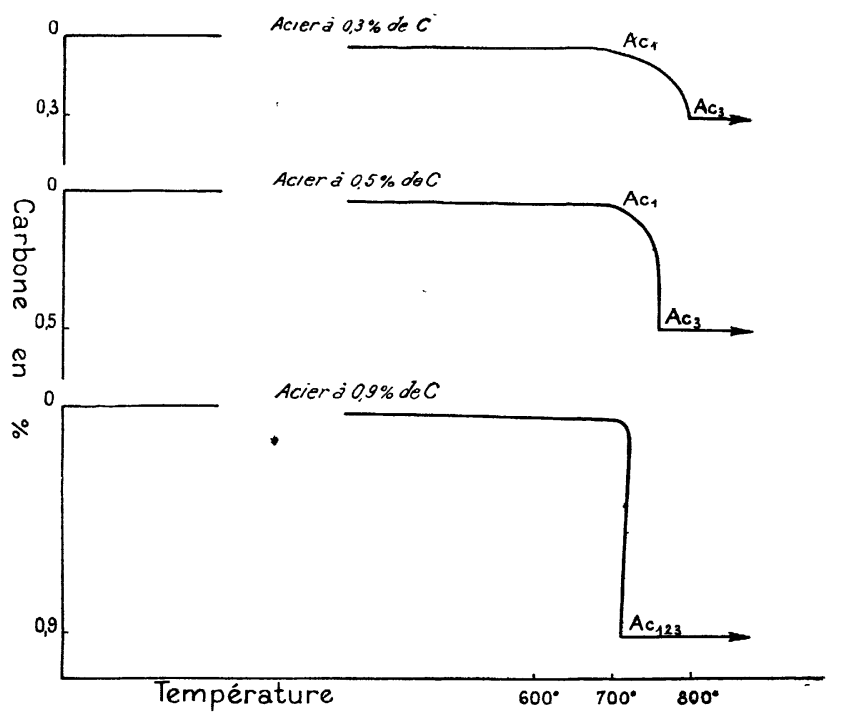

Fig. 6. - Variation de la teneur en carbone (ou en $\mathrm{Fe}^{3} \mathrm{G}$ ) de la solution solide des aciers, en fonction de la tempéralure.

(D'après J. Seigle.)

M. Seigle a, du reste, illustré sa manière de voir en traçant pour divers aciers, la variation de la teneur en carbone (ou en $\mathrm{Fe}^{3} \mathrm{C}$ ) de la solution solide des aciers, en fonction de la température. Ce sontles courbes que nous reproduisons fig. 6 . Il ressort nettement de ces courbes aussi bien que du texte mème, énoncé plus haut, que cet auteur admet que les points $\mathbf{A c}_{1}$ et $\mathrm{Ar}_{1}$ marquent l'un le début, l'autre la fin de la transformation progressive $x \rightleftharpoons \gamma$ du fer et qu'il ne devait se passer en ce point aucune discontinuité brusque, puisque ce n'est pas là que la perlite se formerait ou se décomposerait.

M. Seigle insiste du reste sur ce point et le précise $\left(^{1}\right)$ en se basant sur l'examen des anomalies magnéliques d'acier dur à plus de 0,40 à 0,45 pour 100 de carbone. (Il choisit à dessein de tels aciers pour lesquels les points $A_{2}$ et $A_{3}$ étant confondus l'interpétation des résultats pourra être plus simple).

D'après la théorie classique, dit-il : “ On devrait s'attendre à une augmentation lente de magnétisme depuis $A r_{3}$ jusqu'à Ar ${ }_{1}$, puis une augmenlation brusque quant on passe au refroidisssement par le point $\mathbf{A r}_{1}$.

"A l'échauffement, les phénomènes étant inverses, on devrait sattendre à une chute de magnélisme quand on

(1) Yoir : Génie Cicil, 21 octobre 1934, p 400. 
passe par $\mathrm{Ac}_{1}$. On aurait ainsi des tracés tels que celui de la figure $\%$. Or l'expérience ne montre rien de semblable; il n'y a notamment, aucun changement brusque semblabte au point Ar $r_{1}$ considéré dans la conceptionclassique comme correspondant à la formation de la perlite. ")

Nous ne pouvons adopter cette manière de voir car nos expériences personnelles sont en contradiction absolue avec celles sur lesquelles s'appuie M. Seigle.

Il dit que si la théorie classique était exacte nous devrions avoir une augmentation lente de magnétisme depuis $\mathrm{Ar}_{3}$ jusqu'à $\mathrm{Ar}_{1}$. Au chauffage, les phénomènes devraient se produire en sens inverse. Or, c'est exactement ce que l'on obtient.

Nous reproduisons ci-après (fig. 9) les courbes que nous avons publiées il y a 13 ans dans une étude expé-

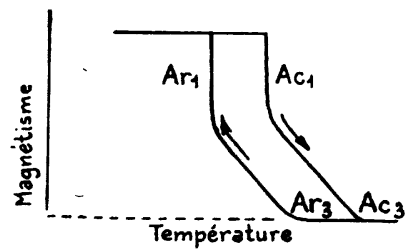

Fig. 7. - Représentation des changements de magnétisme d'un acier dur hy poeutectoïde si la conception classique est exacte.

(D’après J. Seigle).

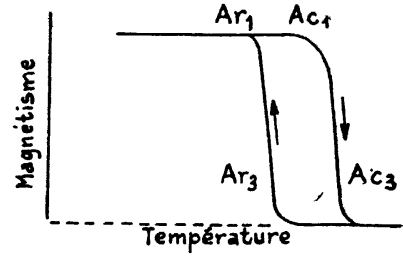

Fiq. 8. - Tracé observé (D iaprès J. Seigle )

Variation de l'induction en fonction de la température : acier à 0,40 pour 100 de carbone placé dans un champ $H=20$ gau-s.

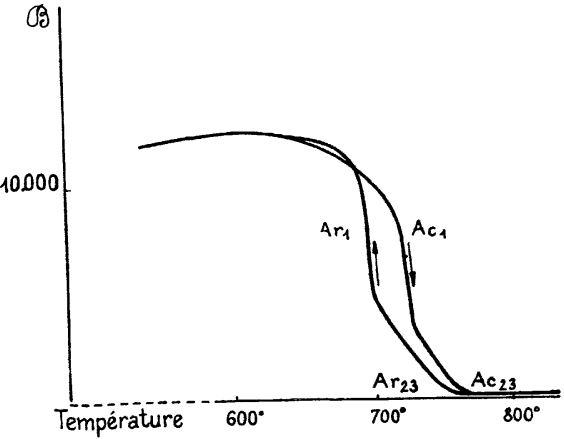

Fig. 9. - Courbes obtenues avec un barreau cylindrique.

$d=3 \mathrm{~min} ; \quad l=200 \mathrm{~mm}$

$\bar{l}=-166,6$.

Du reste des courbes en tous points identiques ont été obtenues par Kotaro Honda $\left.{ }^{1}\right)$.

Dans un cas comme dans l'autre nous arons une chute brusque du magnétisme au point $A c_{1}$, puis une diminution progressive entre $\mathrm{Ac}_{1}$ et $\mathrm{Ac}_{2}$ ou $\mathrm{Ac}_{23}$, suivant la teneur en carbone de l'acier.

D'où vient alors la méprise de $M$. Seigle ?

Elle est assez facile à expliquer. Les courbes qui font l'objet de notre figure 9, ont été obtenues avec un barreau cylindrique ayant une longueur égale à 66,6 fois le diamètre, c'est-à-dire avec un échantillon dans lequel le champ démagnétisant était très faible. Or nous avons montré dans le mémoire précédemment cité, que si nous étudions la chute du magnétisme en fonction de la température sur le même acier que précédemment mais avec un barreau cylindrique dont la longueur n'est plus que כ̆ fois le diamètre, c'est-à-dire dans İequel le champ démagnétisant est très important

(i) K. Hoxda. On the $A_{2}$ line in the équilibrium Diagram of the Iron-carbon system $=$ Science reports of the Tôhuku tmperial Uni $i$ versity. 1926, série 1, vol. 15, No 2, p 248.

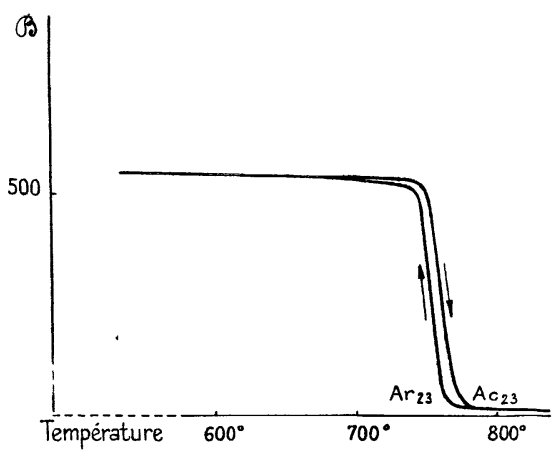

Fig. 10. - Courbes obtenues avec un barreau cylindrique.

$d=11,3 \mathrm{~mm} ; \quad l=56 . .3 \mathrm{~mm}$;

$\frac{d}{l}=5$.

nous obtenons alors les courbes de la figure 10 tout à fait analogues à celles de $\mathbf{M}$. Seigle reproduites figure 8 . Et il se trouve en effet que cet auteur a opéré avec le thermomagnétomètre de Chevenard dans lequel on utilise de petits barreaux $(d=2 \mathrm{~mm}, l=10 \mathrm{~mm})$ c'est-à-dire dont la lougueur est égale à $7, \ddot{b}$ fois le diamètre et par conséquent, à très grand champ démagnétisant. Il n'est donc pas élonnant qu il ait obtenu des courbes analogues à c:elles de notre figure 10 et n'ait pas aperçu la chute brusque de magnétisme qui se produit au point $A c_{1}$. Mais, la seule faulive est la mélhode expérimentale et, il n'y pas lieu de rejeter pour cela la théorie classique de la formation de la perlite. D'autant p'us que bien d'autres expériences viennent confirmer cette théorie.

Ainsi, nous donnons figure 11 une série de courbes dedilatation, en fonction de la température, obtenues au dilatomètre Pellin, sur des fers et aciers à teneur en

(1) P. Deseax. Etude expérimentale sur les transformations. magnétiques du fer et des aciers. Annales de Physique, 1922, 9 e série, t. 23, p. 17 !. 
carbone variable. On notera en passant la très grande différence qui existe entre la courbe de dilatation du fer électrolytique et celle d'un acier extra doux à 0,14 pour 100 de carbone. On ne peut done pas prendre indifféremment l'une ou l'au tre comme semble l'indiquer M. Seigle.

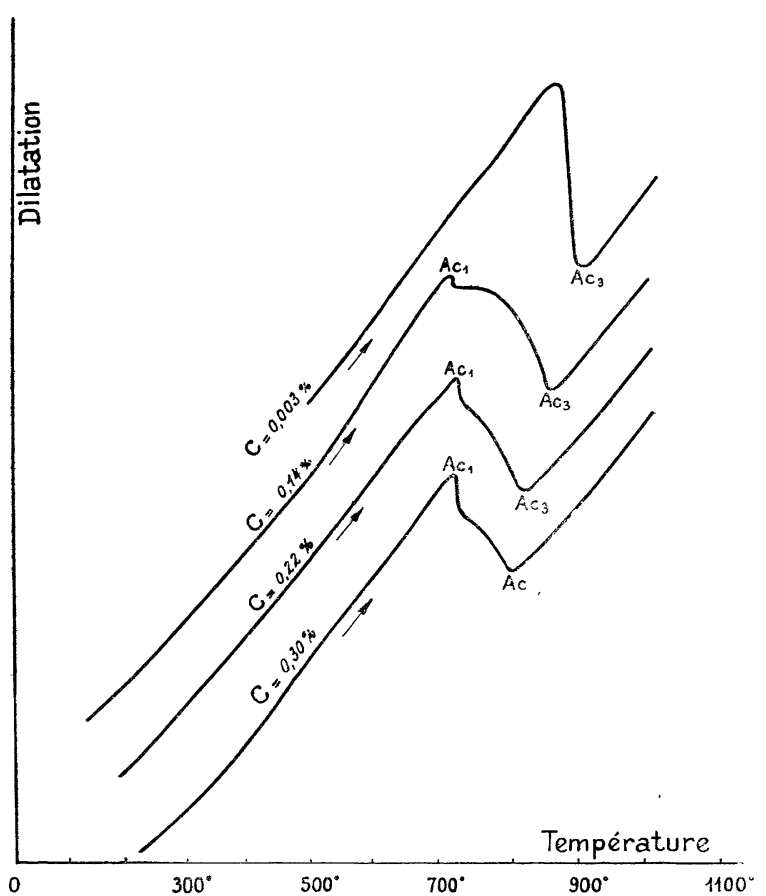

Fig. 11. - Courbes de dilatation de divers aciers en fonction de la température de chauffage.

En outre, nous trouvons sur ces courbes, au point $A c_{1}$, une anomalie de dilatation pour tous les aciers contenant un peu de carbone et dont l'intensité croît avec la proportion de perlite.

Ce résultat expérimental s'explique très bien avec la théorie classique qui admet que la perlite se forme au point $\mathrm{Ar}_{1}$ et se redissout au point $\mathrm{Ac}_{1}$. En revanche, on ne voit pas comment pourrait s'expliquer cette brusque anomalie dilatométrique au point $\mathrm{Ac}_{1}$ si on admettait avec M. Seigle que ce point marque simplement le début de la transformation progressive du fer $\alpha$ en fer $\%$.

Les mêmes conclusions s'imposent si on regarde les courbes de refroidissement obtenues par la méthode différentielle de Roberts-Austen, avec l'appareil Saladin Le Chatelier, sur une série d'aciers au carbone (fig. 12).

Il est impossible d'expliquer l'anomalie d $\mathbf{u}$ point $\mathbf{A r}_{1}$ si on admet comme M. Seigle que la perlite ne se forme pas en ce point $\mathrm{Ar}_{1}$.

$3^{\circ}$ Objection. - On ne peut assimiler les transformations de la solution solide $\%$ (austénile) des aciers, et la formation de la perlite, à celle de deux métaux fon. dus $A$ et $B$ donnant un eutectique par leur solidification.
"Cette assimilation n'est pas correcte ", dit M. Seigles: “ dans le cas des métaux, on a deux constituants $A$ et $B$, "qui sont les deux métaur; dansle cas des aciers, on a "l'équivalent de trois constituants, à savoir: le fer $\gamma$, le

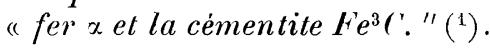

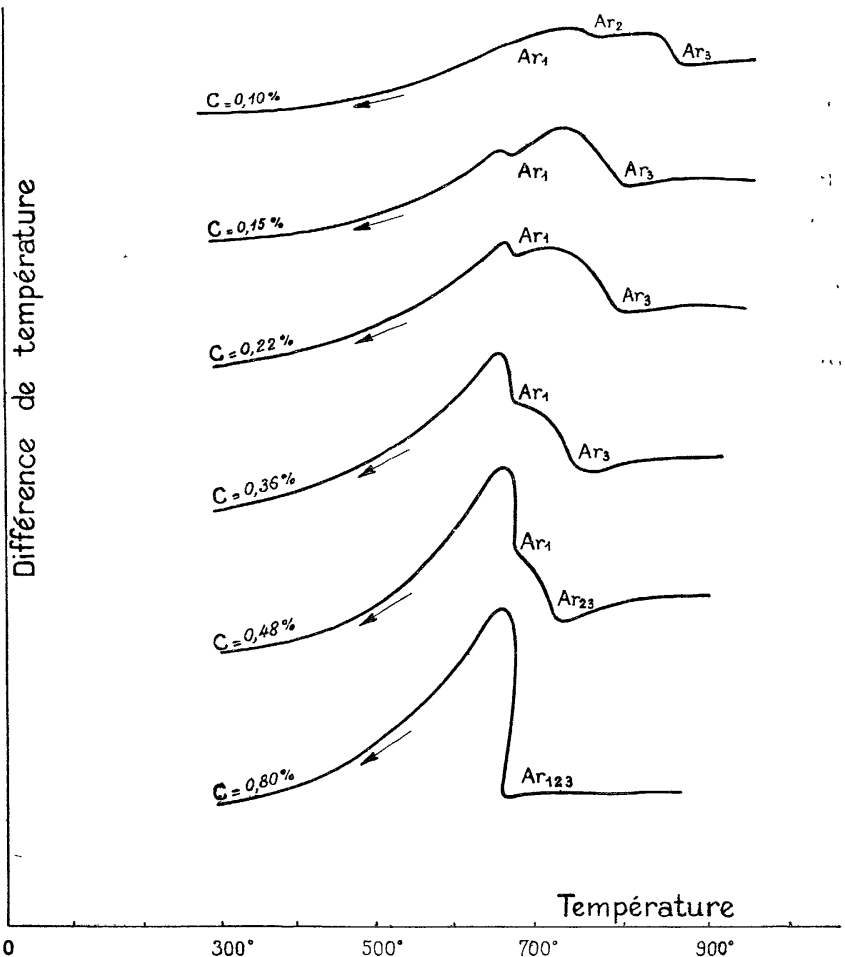

Fig. 12 - Courbes de refroidissement de divers aciers obtenues par la méthode différentielle de Roberts-Austen arec l'appareil Le Chatelier-Saladin.

Avant de répondre à cette objection, il est nécessaire de rappeler un cerlain nombre de notions.

$1^{\circ}$ Dans un alliage en équilibre, un seul corps (métal pur ou combinaison chimique) ne peut jamais être compté que comme un seul constituant, quel que soit son état (solide, liquide ou gazeux), même sil y a coexistence simultanée de plusieurs de ces phases. ll en est également ainsi des variétés allotropiques d'un mème corps qui ne donnent jamais qu'un seul constituant: mais qui constituent cependant autant de phases distinctes.

$2 \circ$ Etant donné le peu d'importance de la phase gazeuse dans les réactions d'équilibre en métallurgie, on fait généralement, sous certaines réserves, abstraction de cette phase.

$3^{\circ}$ On doit noter encore, que dans les alliages binaires l'un des métaux peut être remplacé par une combinaison. Tel seraitle cas des alliages de $\mathbf{M g}$ et $\mathbf{M g ~} \mathbf{Z n}^{2}$ dans lesquels les constituants indépendants ne sont pas $\mathbf{M g}$

(1) Seigre. Nouvelle théorie proposée pour la formation des aciers recujts. Le Genie Civil, 14 octobre 1933, t. 103, p. $37 \theta$, ligne 28. 
et $\mathbf{Z n}$; mais bien $\mathrm{Mg}$ et $\mathrm{MgZn}^{2}$. Tel est aussi le cas des aciers, où les constituants indépendants ne sont pas le fer et le carbone, mais bien $\mathrm{Fe}$ et $\mathrm{Fe}^{3} \mathrm{C}$.

Ceci étant admis, on necomprend pas bien l'objection de M. Seigle. Il oppose le cas de deux métaux donnant un eutectique par leur solidification au cas des aciers composés de Feet de $\mathrm{Fe}^{3} \mathrm{C}$ qui forment l'eutecioïde perlite.

Il dit que dans le cas des deux métaux $\mathrm{A}$ et $\mathrm{B}$, on a deux constituants : le métal A et le métal B, ce qui est parfaitement exact. 11 pourrait même ajouter que, pendant la solidification de l'eutectique, on a en présence : $1^{\circ}$ la partie encore liquide qui est une solution liquide des deux métaux l'un dans l'autre, et qui ne constilue qu'une phase, et $2^{\circ}$ la partie solide qui est formée de l'eutectique déjà déposé et qui se compose de métal A juxtaposé au métal B, soit deux phases. Ce qui fait un total de 3 phases; une liquide et deux solides.

Dans le cas des aciers, au moment de la formation de l'entectoïde, perlite, nous n'avons pas trois constituants comme le voudrait. M. Seigle mais seulement deux, car si le fer $x$ et le fer $\gamma$ sont deux phases distinctes, ils ne sont pas deux constituants différents. Nos deux constituants sont donc d'une part le fer et d'autre part le carbure de fer. Lors dela formation de la perlite nous avons en présence : de l'austénite, solution solide de fer $\gamma$ et de carbure de fer (une phase) qui se décompose en deux phases (fer $\alpha$ et carbure de fer) soit trois phases en tout et seulement deux constituants.

Nous sommes donc bien dans un cas absolument parallèle à celui de laformation de l'eutectique de deux métaux, ou si on veut être plus précis, à l'eutectique d'un métal et d'une combinaison, tels par exemple $\mathrm{Mg}$ et $\mathrm{MgZn}$.

On peut par conséquent faire rigoureusement et d'une manière très correcte l'assimilation incriminée par M. Seigle.

Conclusion. $-1^{\circ}$ La transformation $\alpha \Longleftarrow \gamma$ du fer pur ne s'effectue pas d'une manière étalée entre les points $A_{1}$ et $A_{3}$; mais bien uniquement au point $A_{3}$.

$2^{\circ}$ La perlite a une composition constante et sa formation se produit au point $\mathrm{Ar}_{1}$, conformément aux lois qui régissent la formation des eutectoïdes, rien ne s'oppose donc à la considérer comme tel

$3^{\circ}$ La théorie dite " théorie classiqne » du diagramme $\mathrm{Fe}-\mathrm{Fe}^{3} \mathrm{C}$ n'a été mise en défaut, jusqu'ici, par aucun fait expérimental nouveau. Il n'y a donc pas lieu, pour linstant, de lui apporter le moindre changement. 


\section{Réponse de M. Seigle aux objections de M. Dejean.}

J'ai lu avec beaucoup d'intérèt l'éxposé de M. Dejean, attaché aux conceptions classiques; je rappellerai cependant quelques constatations expérimentales qui montreront tout au moins qu'il y a encore beaucoup de choses à comprendre qui ne sont pas simples.

Région troublée à l'échauffement du fer entre des températures de l'ordre de 700 à 920 '. Il y a un fait inconlestable, c est que les fers et aciers les plus purs que nous sachions préparer présentent une région troublée entre $701^{\circ}$ (ou moins) et 920 à $930^{\circ}$ environ à l'échauffement, et il y a de même une région troublée au refroidissement, à des températures un peu moindres, par suite de l'hystérésis.

Difficultés d'interprétation. - Il y a malheureuscment plusieurs ordres de difficultés dans les conclusions à tirer des observations, et je citerai les suivantes :

$1^{\circ}$ Les diverses méthodes d'étude ne caractérisen 1 pas exactement de même la région troublée.

2. Beaucoup de courbes exprimant une proprièté physique en fonction de la température ont été élablies par des expérimentateurs seulement pour le cas de l'échauffement; or, des courbes pour le cas du refroidissement seraient très intéressantes, afin de préciser l'importance et l'étalement de l'hystérésis.

Il conviendrait, nolamment. d'établir des courbes pour des refroidissements qui débuteraient dans la période troublée, et non pas seutement une fois celle ci entièrement terminée.

$3^{\circ}$ Il convient de se demander si les échantillons les plus près du fer à 100 pour 100, - par exemple 94, 8 ou 99,9 pour $100,-$ n'ont cependant pas leurs propriétés modifiées soit par les faibles impuretés reslantes, soit par certain manque d'homogénéité dans une certaine direction par rapport aux autres directions.

Le fer Armco et le fer électrolytique sont les deux qualités les plus proches du fer pur que l'on sache maintenaut préparer d'une façon courante; le fer Irmco contient environ 99,85 pour 100 de fer et le fer électrolytique atteint à 99,96 pour 100 .

Or, dans les deux cas interviennent des gaz dissous ou occlus, et d'une façon qui nous échappe; mais des développements à ce sujet dépasseraient le cadre de cette réponse. Je rapporterai plus loin sommmairement quelques essais dilalomélriques très curieux.

Rappel de quelques constatations expérimentales autres que par la méthode dilatométrique.

a) Chute de l'aimantaticn spécifique $\sigma$ à l'échauffement. quand on arrive au point de Curie. - Cette chute se présente assez brusquement vers $770^{\circ}$ dans un champ faible (par exemple 30 gauss) mais elle est assez progressive dans 11 champ fortde $10 \% 11$ gauss et plus.

b) Courbe du paramagnétisme, (valeur de $\frac{1}{y}$ en fonction de la température). - Les auteurs qui ont fait des mesures n'ont pas trouvé exaclement les mêmes chiffres c'est-à-dire n'ont pas établi exactement les mêmes courbes, ce qu'on peut attribuer à une non-identité parfaite des échantillons éludiés.

J'ai reproduit dans la figure 14 de mon étude du.Journal de Physique de janvier 1934, le tracé représentant les déterminations deMM. Weiss et Foëx ; ils en concluaient l'existence entre $770^{\circ}$ (point de Curie) et 900 environ (rlébut de l'état $\gamma$ ) d'un fer $\beta_{1}$ à 12 magnétons par atome, puis l'existence d'un fer $\beta_{2}$ à 10 magnétons; ces deux variétés $\beta_{1}$ et $\beta_{2}$ pouvant d'ailleurs ne pas diflérer allotropiquement du fer $\alpha$.

Mais comme les déterminations d'autres expérimentateurs sont un peu différentes, l'existence de ces variétés $\beta_{1}$ et $\beta_{2}$ reste douteuse.

c) Thermo résistivité. - La ligure 1 reproduit le tracé obtenu par M. Chevenard (') sur du fer électrolytique, avec la courbe dérivée.

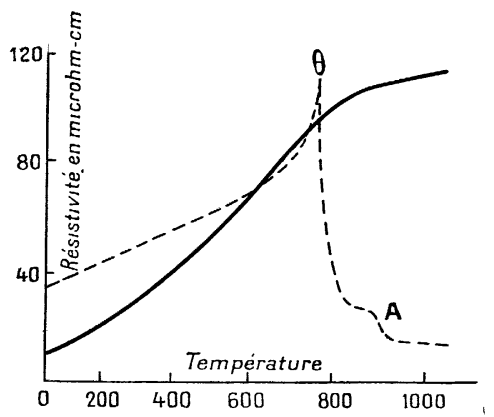

Fiğ. 1.-Thermorésistivité du fer électrolytique et courbe dérivée, d'après $M$. Chevenard

$0=$ point de Curie de fer $\alpha$.

$A=$ transformation allotropique $x \rightarrow \gamma$.

Etant donné la position de l'anomalie constatée vers $900^{\circ}$, il s'agit certainement d'un tracé à l'échaulfement. Il serait évidemment très intéressant d'avoir un tracé au refroidissement, pour constater comment l'hystérésis du phénomène se présente.

d) Pouvoir thermoálectrique. - La figure 2 reproduit le tracé obtenu par M. Cherenard $\left({ }^{2}\right)$. Il y a un

(') Reıue du N'ickel, $3^{*}$ année, $n^{\bullet} 2$.

(“) Revue du Nirtiel, 3" année, n². 
premier changement de direction altribué au point de Curie $\theta$ ou point $\Lambda_{2}$ et un nouveau changement attribué au point $A_{3}$; il s'agit encore uniquement d'une courbe à l'échauffement.

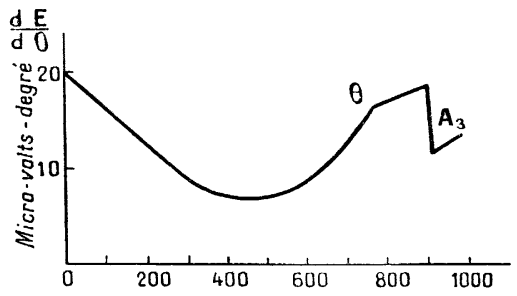

Fig. 2. - Courbe pouvoir thermo-électrique-température du fer pur, d'après $\mathrm{M}$. Chevenard.

Tracés dilatométriques. - J'avais voulu, dans la Iigure 1 demon étudedu Journalde Physique, - janvier 1934 - montrer la correspondance entre dilatation réelle et dilatation différentielle, prises comme ordonnées, en fonction des températures prises comme abcisses, et je déformai malheureusement les tracés - en les arrondissant trop dans la région d anomalie - afin de rendre cette correspondance plus claire ; c'cst. cela qui motive très justement l'observation de M. Dejean .

Mais il est certain que les différents expérimentateurs n'ont pas obtenu exactement les mêmes tracés, et il y a soit différence d'allure, soit différence dans la température moyenne de la portion importante de l'anomalie.

Le point imporlant est celui-ci : a-t-on une anomalie dilatomélrique étalée en température ou non élalée?

Supposons qu'on ait une anomalie non étalée se produisant à la température $T$ par suite d'une transformation allotropique, et supposons en outre, pour simplifier, que le coefficient de dilatation soit constant en fonction de la température pour chacun des deux états.

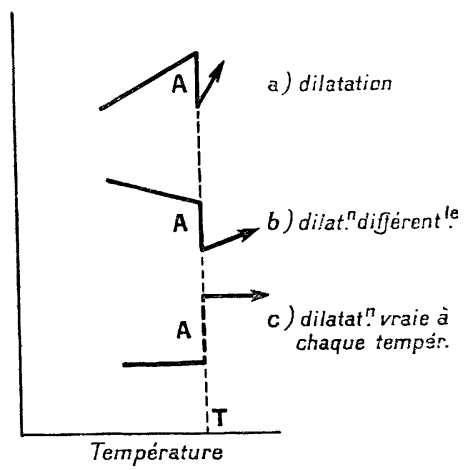

Fig. 3. - Courbe schématique des effets d'une transformation $A$ à la lempérature $T$ pour trois types de représentalion de la dilatation en fonction de la température.

Cela correspond aux schémas des figures $(3 a, b, c)$ limités à la région anormale :

(1) Voir Borelixs, p $\{00$ el Oberhoffer, p. 24 a) Dilatation-température ;

b) Dilatation différentielle-température;

c) Dilatation vraie à chaque température en fonclion de la température.

Or, je constate d'abord que les tracés rapportés par M. Dejean d'après M. Bouchayer et d'après $M$. Sato, présentent, pour la partie importante des anomalies, un ÉTALEMENT SUR UNE TRBNTAINE DE DEGRÉS AU MOINS, tant à l'échaựfement qu'au refroidissement.

Mais voici encore deux tracês d'autres expérimentateurs.

Tracé de $M$. Benedicks cité par Oberhoffer ('). La figure 4 reproduit le tracé de dilatation différentielle sur un barreau à 99,967 pour 100 de fer; c'est sans doute du fer électrolytique;les ordonnées sont à une très grande échelle.

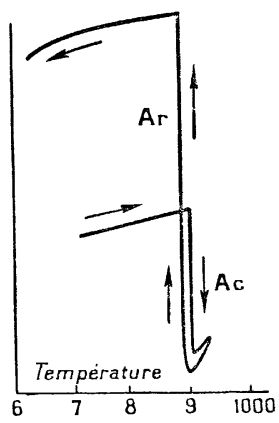

Fig. 4. - Courbe dilatométrique différentielle du fer pur à $\subseteq 9,967$ pour 100 , d'après M. Benedicks, à une grande échelle des ordonnées.

A l'échauffement, il y a une forte contraction anormale très peu étalée - sans doute entre 905 et $915^{\circ}$.

A $u$ refroidissement, la dilatation anormale a une valeur beaucoup plus grande. Le barreau revenu à la température ordinaire est donc plus long qu'au départ. Il n'est pas donné d'explication à ce sujet; mais j'ai eu moi-mème des tracés semblables dans les conditions indiquées ci-après. Le tracé de M. Benedicks montre de

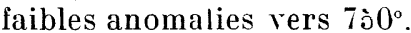

Tracés de $M$. Chevenard. - Les tracés reproduits figures 5 et 6 sont relatifs à du , fer électrolytique refondu au four Tammann s; j'ignore le détail de la fusion, de la coulée et de la préparation des barreaux.

Dans la figure $\tilde{5}$, qui est encore un diagramme de (dilatation différentielle-température). j'ai tracé pour l'anomalie $\mathrm{A}_{c}$, les tangenles à $60^{\circ}$ dont les points de contact $H$ vers $875^{\circ}$ et $B$ vers $930^{\circ}$ marquent respectivement le point haut et le point bas de la courbe (dilatation réelle-température). Cette anomalie est bien plus étalée que dans le tracé de M. Benedicks.

D'autre part, M. Chevenard a déduit de ses tracés la

(1) Voir son ouvrage " das technische Fisen n, p. 14, figure 6 . 


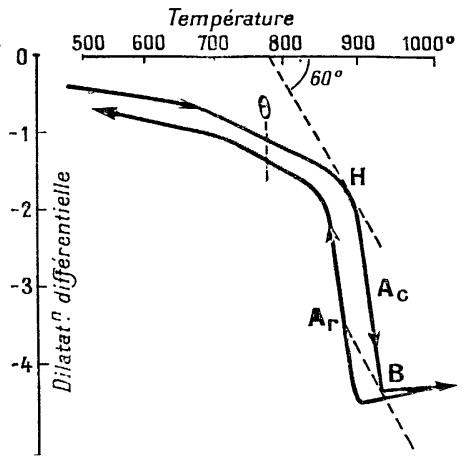

Fig. 3. - Courbe de dilatation différentielle du fer électrolytique fondu au four Tammann, d'après $\mathbf{l}$. Chevenard.

courbe de dilatibilité vraîe du fer en fonction de la température ; voir figure 6 ; elle est très différente $d u$ schéma figure $3 c$.

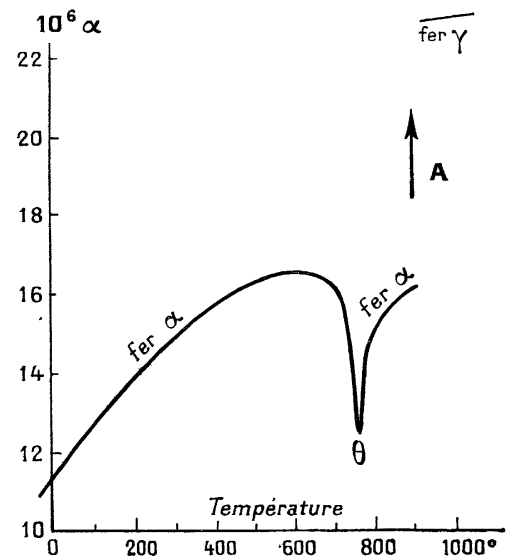

Fig 6. - Dilatabilité vraie du fer pur en fonction de la température, d'après $M$. Chevenard.

$\theta$, point de Curie de fer $\alpha$.

$A$, passage du fer $\alpha$ au fer $\gamma$.

Rappel de quelques-uns de mes tracés dilatométriques (1). - $1^{\circ}$ Fer Armco. - A l'état de barreau prélevé dans un rond laminé de $10 \mathrm{~mm}$ et après un premier recuit dans le dilatomètre - jai bien obtenu le mème tracé que celui de M. Chenevard de la figure $ّ$. Si l'on inscrit plusieurs cycles successifs sur le mème papier, on constate, figure 7 , que le point extrême $F_{1}$ vient successivement en $F_{2}, F_{3} \ldots F_{n}$ dans la suite des six refroidissements et chauffages. Un recuit de 48 heures dans le vide à $850^{\circ}$ ou $900^{\circ}$ rend la forte contraction beaucoup plus brusque, mais elle se présente à une température moyenne un peu plus forte ; la petite anomalie vers $70^{\circ}$ sur le diagramme de dilatation différentielle est rendue plus nette.

(1) Voir plus de détails :

Génie Civil, 12 octobre 1929, 5 juillet 1930 ,

Revue de Metallurgie, mai, juin 1932.

La Technique Moderne, $\mathrm{n}^{\circ} 3,1934$.
Ce qui modifie surtout les tracés, ce sont les recuits dans l'hydrogène, de 2 jours ou plus, à $850^{\circ}$ ou plus.

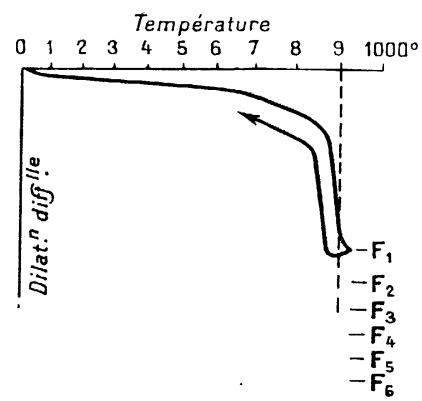

Fig. 7. - Fer Armco-cycles dilatométriques successifs.

La figure 8 en est un exemple. Comme dans l'essai de M. Benedicks de la figure ${ }^{\pi / 4}$, la*dilatation anormale

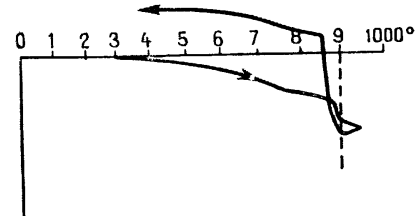

Fig. 8. - Fer Armco et fer électrolytique chauffés $21 / 2$ jours à $1000^{\circ}$ dans l'hydrogène.

au refroidissement est bien plus grande que la contraction anormale à l'échauffement, et le barreau est plus long après chaque cycle.

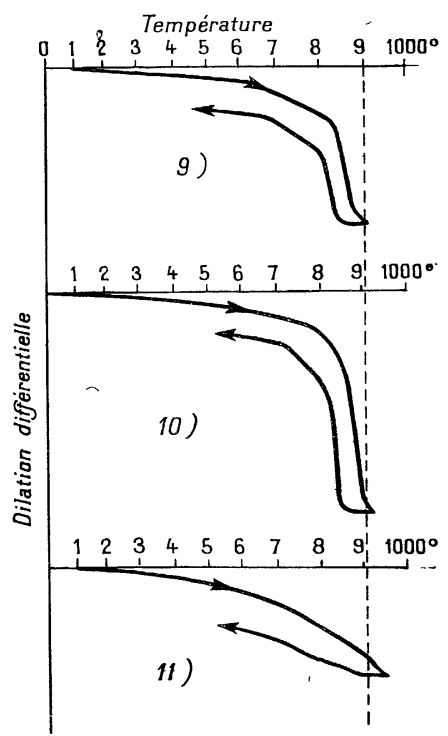

Fig. 9, 10, 11. - Mème acier doux sans perlite. y) Rond laminé de $5 \mathrm{~mm}$ recuit normalement.

10) - - après torsion au rouge de 700 à 11) - $\quad$ - après recuit de 3 jours à $850^{\circ}$ dans l'hydrogène ou dans le vide. 
$2^{\circ}$ Acier doux Thomas, sans perlite, laminé en rond de $5 \mathrm{~mm}$. - Les figures 9, 10 et 11 suffiront pour montrer les étonnants changements qu'on peut obtenir,

Figure 9, rond tel quel ;

Figure 10, rond soumis à une très forte torsion au rouge (700 à 800 tours sur $50 \mathrm{~mm}$ de longueur).

Cette forte torsion - qui n'a rien pu changer à la composition chimique - produit, sur un barreau cassé à froid, une texture spéciale dénotant l'existence dans chaque section d'une orientation de quelque chose - (grains, impurelés. etc. ?) - en forme de cercles concentriques.

Figure 11, rond soumis à un recuit de trois jours dans l'hydrogène à $80^{\circ}$. Un recuit de 2 à 3 jours dans le vide clonne à peu près la même chose.

Résumé général de ces essais. - Bien des choses nous échappent donc et je ne vois pas, pour ma part, lans quelle expérience on peut se dire plus près du cas idéal du fer chimiquement pur, et constitué en une masse que l'on pourrait considérer comme homogène parce qu'elle serait formée d'une infinité de grains voisins sans aucune orientation privilégiée.

Courbes magnétiques en fonction de la température pour des aciers ordinaires carburés, hy poeutectoïdes. - Les tracés de M. Dejean sont très intéressants. Jen'avais pas dans mes dossiers son étude des Annales de Physique, mais seulement son étude présentée au Congrès de Liége de 1932 sur les «Procédés et appareils servant à la détermination des points critiques ». L'influence signalée par M. Dejean de la dimension des échantillons se manifeste bien aussi avec le thermo-magnétomètre de $M$. Chevenard; mais les résultats de $M$. Dejean sont tous, me semble-t-il, relatifs à l'induction dans un champ alternatif. Or, il intervient alors des phénomènes de magnétisme rémanent très compliqués:

L'interprétation du diagramme eutectoïdedoitelle être modifiée? - Tel est le titre de l'étude de M. Dejean, et il conclut, en ce qui le concerne, «qu'il n'y a pas lieu de lui apporter le moindre changement. " Or, on est bien plutòt amené à dire: « Comment faut-il modifier la théorie classique pour la faire cadrer avec les faits?" Je constate, en effet, que presque tous les expérimentateurs - et notamment en Angleterre se sont efforcés d'imaginer telle ou telle retouche à la théorie eutectoïde, telle ou telle influence accessoire, pour expliquer les faits constatés. D'ailleurs, chaque auteur a sa théorie, et aucune d'elles n’a, jusqu'ici, recueilli une adhésion générale.

Conséquence générale de ce qui précède. Le problème de la solubilité du carbone dans le fer, en fonction de la température, reste donc celui-ci : on doit évidemment imaginer qu'il y a une courbe dé solubilité du carbone dans le fer $\alpha$ pur et qu'il y en a également une dans le fer $\gamma$ pur.

Si la transformation $\alpha \rightarrow \zeta$ se fait à une température donnée, sans aucun étalement, nous avons à faire au point $G$ du diagramme classique, et il y a beaucoup de chances pour que le domaine G P S du diagramme soit un domaine solution solide $\gamma+$ ferrite $\alpha$. Mais si la transformation $\alpha \rightarrow \gamma$ est tant soit peu étalèe, serait-ce seulement de $20^{\circ}$ à $30^{\circ}$, c'est donc que nous n'avons plus de point $G$; que nous avons dans cette zone de température un mélange, ou un agrégat, ou une solution solide des états $\alpha$ et $\gamma$, enfin quelque chose de complexe dans lequel la solubilité du carbone devient ellemême plus complexe.

Ce que j’ai supposé, c'est que l'étalement était bien plus grand encore que ces $20^{\circ}$ à $30^{\circ}$, mais le gros du phénomène est tout de même, en général, relativement resserré.

Cas des aciers ordinaires industriels. - Dans nos aciers extra-doux industriels, le total des éléments etrangers - carbone, manganèse, soufre et phosphore, parfois aussi silicium - est notablement plus grand que dans le fer Armco et le fer électrolytique; le pour 100 de fer sera généralement inférieur à 99,50 pour 100; l'étalement de la transformation $\alpha \rightarrow \gamma$ est certainement augmenté.

Le métallurgiste est donc obligatoirement en présence d'un système à base de (fer + impuretés autres que le carbone), lequel pourra ètre à l'état $\gamma$, ou à l'état $\alpha$, ou à des états intermédiaires $(\alpha \gamma)$ selon la température, el ces divers états auront, pour le carbone, des solubilités différentes. 


\section{Réponse de M. Dejean:}

Essais magnétiques. - Je répondrai tout d'abord à l'objection que fait $M$. Seigle au sujet de la détermination de mes courbes induction-température. Il dit que mes essais ont été faits dans un champalternatif et que dans ces conditions il intervient des phénomènes de magnétisme rémanent très compliqués.

J'ai montré dans mon mémoire (1) en quoi le champ alternatif pouvait modifier l'allure des courbes induction-température lorsqu'on oṕrait sur des échantillons de grosse section. Mais iorsqu'un échantillon de faible section est placé dans un champ alternatif de valeur maxima constante et suffisamment importante, ce ne sont pas les phénomènes de magnétisme rémanent qui sont à craindre. Ce qu'il y a de plus probant c'est que les courbes de Curie $\left({ }^{2}\right)$ obtenues par la même méthode que M. Seigle,mais sur échantillon dont la longueur était égale à 62 fois le diamètre (fil de fer à 0,044 pour 100 de carbone) sont absolument identiques à celle que j'ai obtenue sur un acier à 0,06 pour 1100 de carbone. Elles sont absolument différentes d'allure de celles de M. Seigle. Quant aux aciers à diverses teneurs en carbone, j'ai signalé que Kotaro Honda (3) avait oblenu des courbes en tous points identiques aux miennes, bien qu'il n'eût pas opéré dans des champs alternatiis.

En revanche, j'ai obtenu des courbes ayant même allure que celles de M. Seigle et de divers autres expérimentateurs en opérant comme eux avec des échantillons à grand champ démagnétisant.

Ce n'est donc pas ma méthode qui doit ètre incriminée, mais bien l'emploi d'échantillons à grand champ démagnétisant.

Diagramme $\mathrm{Fe}-\mathrm{Fe}^{3} \mathrm{C}$. - Cette mise au point étant faite, revenons au sujet lui-même. M. Seigle reconnaît maintenant que la plupart des expérimentateurs ont trouvé que les courbes dilatométriques relatives aux fers les plus purs présentaient au point $A_{3}$ (transformation) une anomalie assez brusque puisqu'elle ne s'étalait que sur 20 ou $31^{\circ}$. Et il en conclut cependant ceci : « Mais si la transformation $\alpha \rightarrow \gamma$ est tant soit peu étalée, serait-ce seulement de 20 à $30^{\circ}$, c'est donc que nous n'avons plus de point $G\left({ }^{\prime}\right)$; nous avous dans cette zone de température un mélange ou un agrégat ou une solution solide des états $\alpha$ et $\gamma$, enfin quelque chose de complexe dans lequel la solubilité du

(1) P. Dejeas. Etude expérimentale sur les transformations magnétiques du fer et des aciers. Annales de Physique, 192., 9 série, l. 23, p. 171.

(2) P Cunie Propriétés magnéliques des corps à diverses tem-

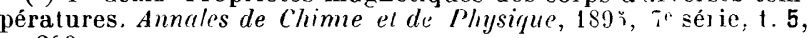
p. 289 .

(3) K. Hoxds. On the A., line in the equilibrium diagram of the Iron carbon system. cience reports of lle Toholit imperial Liniversily, 1926, série 1, vol 12 . nu 2 , p 248.

(4) Il faut comprendre par là que la transformalion ne serait pas isotherme. carbone devient elle-mème plus complexe ". Cette conclusion assez obscure du reste ne correspond pas exactement à la théorie que j’incrimine.

L'étalement sur 20 ou $30^{\circ}$ du point $A_{3}$ peut-il justifier l'hypothèse de $M$. Seigle qui veut que :

“ Dans le domaine intermédiaire de $A_{1}$ à $A_{3}\left({ }^{1}\right)$ existe un état mixte $\alpha \gamma$ à proportions décroissantes de $\alpha$ au chauffage et à proportions décroissantes de $\gamma$ au refroidissement? ")

Il y a là une argumentation bien fragile, alors que ce léger étalement s'explique tout naturellement comme je l'ai déjà dit si on remarque que :

$1^{\circ}$ les fers utilisés, bien que déjà très purs ne sont pas cependant chimiquement purs;

$2^{\circ}$ les vitesses de chauffage et de refroidissement ne sont pas assez lentes pour que l'équilibre soit alteint d'une manière rigoureusement isotherme, surtout dans un métal aussi visqueux que le fer à $900^{\circ}$;

$3^{\circ}$ l'inertie des appareils eux-mêmes joue un rôle qui n'est pas toujours négligeable.

Du reste, la question capitale dans cette controverse est bien plutôt de savoir si oui ou non la perlite est un eutectoïle Si oui. elle doit se former ou se décomposer à température sensiblement constante comme on l'a admis jusqu'ici, c'est-à-dire au point $A_{1}$. Or, les courbes que l'on obtient par les principales méthodes actuellement mises en œuvre pour la recherche des points critiques (méthodes dilatométrique, magnétique ou thermique différentielle) dénotent toutes une anomalie brusque au point $A_{1}$, dont l'intensité est sensiblement proportionnelle à la teneur en perlite de l'acier étudié. L'hypothèse de la perlite eutectoöle est donc entièrement confirmée par ces faits.

Au contraire, il devient absolument impossible d'expliquer l'anomalie $A_{1}$ si on admet avec M. Seigle que ce point ne correspond qu'à la fin d'une transformation progressive étalée entre $A_{3}$ et $A_{1}$ et produite en majeure partie au voisinage de $\rfloor_{3}$.

Je crois donc avoir pleinement le troit de conclure que la théorie dite ( théorie classique » du diagramme $\mathrm{Fe}-\mathrm{Fe}^{3} \mathrm{C}$ n'a été mise en défaut, jusqu'ici, par aucun fait expérimental nouveau et qu'il n'y a donc pas lieu, pour l instant, de lui apporter le moindre changement.

Est-ce à dire que cette théorie restera indéfiniment inchangée? Ce serait de l'enfantillage de le prétendre. Comme toute théorie, elle est sujette à des mollifications et à des retouches. Le champ reste ouvert aux chercheurs. Je suis sur ce point entièrement d'accold avec M. Seigle Je dis seulement qu'aucune des expériences faites jusqu'à ce jour ne permet de prévoir dans quel sens pourront se faire les retouches.

(1) C'est-à-dire de $710^{\circ}$ à $910^{\circ}$. Sergis. Journal de Physique, janvier 1934 , p. 46, 2e colonne, ligne 7 el suivantes. 